\title{
PREDICCIÓN NO LINEAL Y ANÁLISIS DE SERIES TEMPORALES DE VARIABLES MICROMETEOROLÓGICAS MEDIDAS EN EL PANTANAL MATOGROSSENSE
}

\author{
MELLO, Geison Jader - geisonjadermello@gmail.com \\ Instituto Federal de Mato Grosso - IFMT \\ BIUDES, Marcelo Sacardi - marcelo.biudes@gmail.com \\ Universidade Federal de Mato Grosso - UFMT \\ GOMES, Raphael de Souza Rosa - thesivis@gmail.com \\ Univerisdade Federal de Mato Grosso - UFMT
}

\begin{abstract}
RESUMEN: Las variables micrometeorológicas medidas en ecosistemas poseen comportamiento no lineal y representan la dinámica de los procesos físicos y biológicos que ocurren entre la superficie vegetal y la atmósfera. Por lo tanto, el objetivo de este trabajo fue entender el comportamiento micrometeorológico de un bosque estacionalmente inundado en el Pantanal Matogrossense, a partir del análisis de las series temporales de saldo de radiación, temperatura y humedad del aire (con datos de 15 en 15 minutos), medido sobre el pabellón, y hacer predicciones utilizando la teoría de los sistemas dinámicos. Fue aplicado el método de predicción no lineal simple que utiliza una serie medida para estimar una serie prevista, que se comprueba en términos de correlación, exactitud y errores dentro del tiempo establecido por el parámetro de predicción de confianza. Los resultados evidencian la existencia de atractores extraños de baja dimensión fractal y sensibles a la estacionalidad, y que el método de predicción no lineal simple captura la dinámica general de las series temporales micrometeorológicas medidas.
\end{abstract}

Palabras Clave: dinámica no lineal, reconstrucción del espacio de fase, predicción no lineal.

NON-LINEAR PREDICTION AND TEMPORARY SERIES ANALYSIS OF MICROMETEOROLOGICAL VARIABLES MEASURED IN THE MATOGROSSENSE PANTANAL

ABSTRACT: The micrometeorological variables measured in ecosystems have non-linear behavior and represent the dynamics of the physical and biological processes that occur between the plant surface and the atmosphere. Therefore, the objective of this work was to understand the micrometeorological behavior of a seasonally flooded forest in the Pantanal Matogrossense, from the analysis of the time series of radiation balance, temperature and humidity of the air (with data of 15 in 15 minutes), measured on the pavilion, and make predictions using the theory of dynamic systems. Was applied the simple non-linear prediction method that uses a measured series to estimate a predicted series is applied, which is checked in terms of correlation, accuracy and errors within the time set by the confidence prediction parameter. The results show the existence of strange attractors of low fractal dimension and seasonally sensitive, and that the simple nonlinear prediction method captures the general dynamics of the measured micrometeorological time series.

Keywords: nonlinear dynamics, phase space reconstruction, nonlinear prediction.

PREDIÇÃO NÃO LINEAR E ANÁLISE SERIAL TEMPORÁRIA DE VARIÁVEIS MICROMETEOROLÓGICAS MEDIDAS NO PANTANAL MATOGROSSENSE

RESUMO: As variáveis micrometeorológicas medidas em ecossistemas possuem comportamento não linear e representam a dinâmica dos processos físicos e biológicos que ocorrem entre a superfície vegetada e a atmosfera. Sendo assim, o objetivo deste trabalho foi entender o comportamento micrometeorológico de uma floresta sazonalmente inundada no Pantanal Mato-grossense, a partir da análise séries temporais 
de saldo de radiação, temperatura e umidade do ar (com dados de 15 em 15 minutos), medidos acima do dossel, e fazer predições utilizando a teoria dos sistemas dinâmicos. Foi aplicado o método de Predição Não Linear Simples que utiliza uma série medida para estimar uma série prevista, que é verificada em termos de correlação, exatidão e erros dentro do tempo estabelecido pelo parâmetro de previsão confiável. Os resultados evidenciam a existência de atratores estranhos de baixa dimensão fractal e sensíveis à sazonalidade, e que o método de predição não linear simples captura a dinâmica geral das séries temporais micrometeorológicas medidas.

Palavras-Chave: dinâmica não linear, reconstrução do espaço de fase, predição não linear.

\section{INTRODUCCION}

La cuestión de la descripción de los bosques, teniendo como ejemplo los bosques inundados es un problema brasileño extremadamente significativo (GOMES \& VARRIALE, 2004). La búsqueda de la comprensión del comportamiento del ecosistema a través de fenómenos micrometeorológicos y sus interacciones con la superficie terrestre involucra la teoría de los sistemas dinámicos y depende de los fenómenos observados / descritos y su escala espacio-temporal (MELLO et al., 2011; MELLO et al. 2012a). La no linealidad está presente en las características fundamentales que determinan la dinámica del comportamiento de áreas inundadas, también en su interacción con la vecindad terrestre, con la cuenca hidrográfica a la que pertenece, con la atmósfera así como con los efectos antropogénicos (GOMES \& VARRIALE, 2004, MELLO et al., 2012b, MELLO et al., 2013a).

Dada la complejidad del sistema ambiental Pantanal, con su elevado número de variables en interacción, la fundamentación teórica de este trabajo fue dirigida hacia una teoría robusta adecuada a esta aplicación: la teoría de los sistemas dinámicos. Esta teoría está a la vanguardia de la investigación en ciencias ambientales, a ejemplo de la física ambiental, biometeorología y ciencias atmosféricas (MILLÁN et al., 2010).

Sin embargo, antes del enfoque por teoría de los sistemas dinámicos es fundamental que estas series temporales microclimatológicas medidas en torres experimentales sean probadas con respecto a su no linealidad, justificando de forma sólida la aplicación de tales herramientas (MELLO et al., 2012b). Así, en la siguiente Sección se presentó una revisión sobre series temporales lineales y no lineales con foco en la prueba de no linealidad en términos de eficacia y limitación.

En esta perspectiva el objetivo general de este trabajo fue entender el comportamiento micrometeorológico del Pantanal a partir del análisis de las variables micrometeorológicas saldo de radiación, temperatura y humedad relativa del aire medidas cada 15 minutos buscando predecir este comportamiento por medio de la teoría de sistemas dinámicos.

Para ello, se definieron los siguientes objetivos específicos: analizar las series temporales micrometeorológicas, reconstruir los atractores asociados a esas series y analizar sus recurrencias cualitativa y cuantitativamente, estimar el tiempo máximo de predicción confiable y hacer predicciones utilizando el método de predicción no lineal simple. 


\section{PREDICCIÓN NO LINEAL Y VERIFICACIÓN DEL MODELO}

El modelo de predicción utilizado en este trabajo, la Predicción no lineal simple, parte de una serie temporal escalar (pasado) con el objetivo determinar una estimación para sus valores siguientes (futuro), sin que se conozcan las ecuaciones de gobierno del fenómeno físico asociado al mismo, (SAVI, 2006). Uno de los aspectos interesantes de este método es la posibilidad de hacer predicciones sin la construcción explícita de un modelo para una serie temporal específica.

A continuación se presentan los parámetros que involucran la predicción, tales como el tiempo máximo de predicción fiable $\xi$ y los aspectos del método de la predicción no lineal simple.

\subsection{TIEMPO MÁXIMO DE PREVISIÓN CONFIABLE}

El Expo de Lyapunov $\lambda \_$max es una cuantificación del "efecto mariposa" de Lorenz (1963) (FERREIRA, 2009). Por lo tanto, el tiempo máximo de predicción fiable (también llamado "tiempo de Lyapunov"), se justifica a partir de los siguientes supuestos:

Si el atraco no tiene ningún exponente $\lambda_{\max }$ positivo para las $\mathrm{m}$ dimensiones del espacio de fase, o sea, $\lambda_{\max } \leq 0$, entonces el sistema no es caótico; y de esta forma una pequeña perturbación en una órbita hará que ella quede cerca de ésta o haya tendencia exponencial a la cuenca de atracción.

$\mathrm{Si}$ el atraco tiene al menos un $\lambda_{\max }>0$, entonces el crecimiento de las perturbaciones es controlado por $\lambda_{\max }$ lo que impulsa una divergencia exponencial de las trayectorias.

Esto introduce una manera cuantitativa de estimar la previsibilidad del sistema. Así el tiempo máximo de predicción fiable $\xi$ se define como:

$$
\xi=\frac{\tau}{\lambda_{\max }}
$$

Eq. 1

donde $\mathrm{T}$ representa aquí el intervalo de la muestra experimental, $\lambda_{\max }$ el mayor exponente de Lyapunov y el parámetro $\xi$ representa el tiempo máximo de una predicción confiable. Este concepto se ha utilizado recientemente dentro de la perspectiva climática (TSONIS, 2001; GOMES \& VARRIALE, 2004; CHAUDHURI, 2006; MILLÁN et al., 2009).

\subsection{PREDICCIÓN NO LINEAL SIMPLE}

Una forma adecuada para modelado y que tiene en cuenta la no linealidad usando series temporales conocidas del sistema estudiado es la Predicción No Lineal Simple, un método basado en la reconstrucción del espacio de fase. En el caso de que se trate de una serie temporal conocida, llamada Medida $S_{n}$ con $n=1, \ldots, N$, para estimar valores futuros, $S_{n+p}$ con $n=N+$ $1, \ldots, N+p$, llamada Predita. Este modelo establece una forma para estimar las series futuras. Las técnicas de predicción se clasifican, en general, en métodos lineales y no lineales, y también globales y locales. Los principales aspectos relacionados con el análisis de series temporales y predicción no lineal son discutidos y profundizados en Casdagli (1989), Weigend \& Gershenfeld (1994), Schreiber (1999), Kantz \& Schreiber (2004), Savi (2006) y Abarbanel (2006). 
Después de la reconstrucción del espacio de fase adecuado para el desdoblamiento del atractor, se define el radio de sondeo de la hiperesfera $\varepsilon$ que está relacionado con el tamaño de la vecindad $v_{n}$ alrededor del punto $u_{n}$, para realizar la predicción en un instante de tiempo $\Delta n(\Delta n=1, \ldots, p)$ el frente de $N$.

Por lo tanto, para todos los puntos $u_{n}$ cerrados dentro del radio $\varepsilon$ de la vecindad $u_{n}$ se encuentran los puntos de $S_{n}$ (términos de la serie temporal) y sus respectivas predicciones $S_{n+\Delta n}$. La predicción $P_{N+\Delta n}$ se calcula a partir de la media aritmética de las predicciones individuales $S_{n+\Delta n}$. La ecuación de predicción se presenta de la siguiente forma:

$$
P_{n+\Delta n}=\frac{1}{\left|v_{\varepsilon}\left(u_{N}\right)\right|} \sum_{u_{n} \in v_{\varepsilon}\left(u_{N}\right)} S_{n+\Delta n}
$$

Eq. 2

donde $\left|v_{\varepsilon}\left(u_{N}\right)\right|$ es el número de elementos pertenecientes a la vecindad $v_{\varepsilon}\left(u_{N}\right)$. Esto también puede ser interpretado como el promedio de los "futuros" de los vecinos (KANTZ \& SCHREIBER, 2004; VIOLA et al., 2010) (Figura 1).

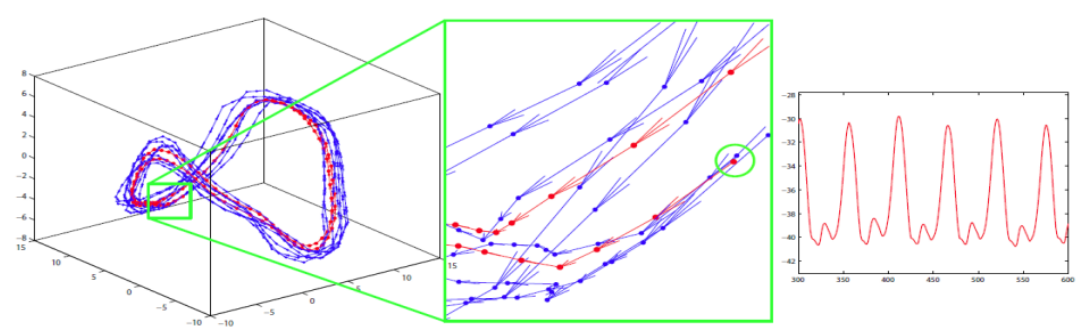

Figura 1 - Esquema de la predicción no lineal simple. Se reconstruye el atrator a partir de una serie temporal medida, y se busca la media del futuro de los estados vecinos. De estos estados futuros se extrae la serie temporal predice (figura adaptada de BASHARAT \& SHAH, 2009).

En el caso de que se trate de un espacio de fase reconstruido en el que todos los vecinos se sondan dentro de un umbral auto-regresivo (del inglés Threshold Autoregresive TAR) para predecir las medidas en el tiempo $\mathrm{N}+\mathrm{P}$ (TONG, 1983; 2007; 2011; 2012); y se clasifica en previsión local en el sentido de que se utilizan términos de una vecindad de radio $\varepsilon$ alrededor de $u_{-} \mathrm{N}$ y no se considera todo el espacio de fase (SAVI, 2006).

Las profundidades sobre las técnicas presentadas en este trabajo se pueden encontrar en Kantz \& Schreiber (2004).

\section{MATERIAL Y MÉTODOS}

\subsection{UBICACIÓN E INSTRUMENTOS}

El sitio experimental se localiza en la Reserva Particular del Patrimonio Natural - RPPN SESC - Pantanal, municipio de Barão de Melgaço - MT, distante 160 km de Cuiabá, Mato Grosso, en la región Centro-Oeste de Brasil, donde está instalada una torre micrometeorológica de $32 \mathrm{~m}$ de altura (16039'50 "S, 56047'50"O, $120 \mathrm{~m}$ ) (Figura 2). Esta zona presenta un bosque monodominante de Cambará (Vochysia divergentes), conocida localmente como cambarazal, con altura del dosel variando entre 28 a 30 m (ARIEIRA \& NUNES DE CUNHA, 2006). El suelo se clasifica como GLEISSOLO HÁPLICO Ta Distrófico. 
El clima es Aw según la clasificación de Köppen, cuya media anual de temperatura del aire es de $25,6^{\circ} \mathrm{C}$ y la humedad relativa del aire es del $73,1 \%$, la precipitación media anual es de $1342 \mathrm{~mm}$, con una precipitación inferior a 15 $\mathrm{mm}$ en el período seco (junio a agosto) y estación lluviosa con el $97 \%$ de la precipitación acumulada anual (BIUDES et al., 2009, INMET, 2009). A menudo la inundación ocurre entre diciembre y marzo $(0,6-1,5 \mathrm{~m}$ de altura) caracterizando la fase ᄀaquática del Pantanal, que acompaña la estación Iluviosa y la oscilación anual del nivel de agua del río Cuiabá, siendo altamente influenciada por la precipitación local (NUNES DA CUNHA \& JUNK, 2004; ARIEIRA \& NUNES DA CUNHA, 2006).
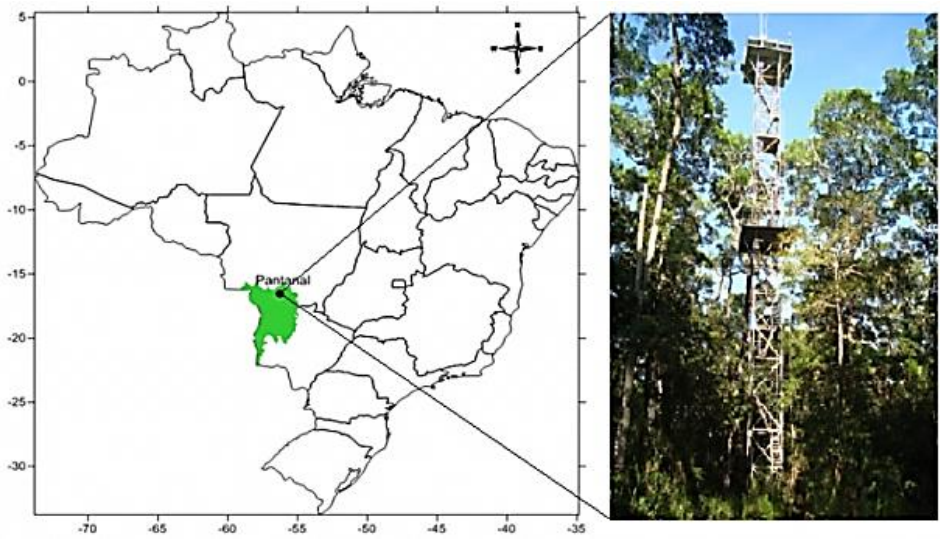

Figura 2 - Mapa del Brasil, con destino a Pantanal Brasileiro, ubicación del área de la vegetación monodominante del cambio en el RPPN SESC y la torre micrometeorológica (Mello et al., 2013b).

Una instrumentación para una columna de datos de saldo de radiación (Rn) para un Radiómetro Net, Kipp \& Zonen Delft, Inc., Holanda; para a temperatura ( $\mathrm{T}$ ) y umidade relativa (Rh) um termohigrômetro (HMP $45 \mathrm{C}$, Vaisala, Inc., Helsinki, Finlandia) para a 37,7 m. Los datos de los procesados y los datos de los registradores (CR 10X, Campbell Scientific, Inc., Ogden, Utah, EE. UU.). Para aumentar el número de canales de entrada no hay información disponible sobre la placa multiplexadora (AM16 / 32A-ST-SW, Campbell Scientific, Inc., Ogden, Utah, EE. UU.).

Los datos utilizados en este trabajo se muestrearon cada 10 segundos con extracción de promedios cada 15 minutos, del 1 de septiembre de 2006 al 1 de septiembre de 2010.

\subsection{ANÁLISIS Y PREDICCIÓN DE SERIES TEMPORATIVAS NO LINEALES}

Analice el tiempo real de las actividades micrometeorológicas: el saldo de la radiación $(R n)$, la temperatura del aire $(T)$ y la umidad relativa $(R h)$ colectada en el sitio experimental.

El programa surrogates.exe del paquete de software TISEAN - Análisis de series de tiempo no lineales (software de dominio público disponible en http://www.mpipks-dresden.mpg.de/ tisean/) para uso para pruebas y no linealidad de las temporadas temporales utilizadas. 
Los cálculos de parámetros necesarios para la reconstrucción de los datos temporales de Informaciones Mutua MI para el tiempo de desfasaje T e Falsos Vecinos Próximos FNN para un máximo de personas en el mercado de la ciudad de Falsos Vecinos Próximos. No te preocupes por la calidad de los gráficos de Recurrencia RP, El tema de los aspectos cuantitativos de las herramientas de análisis de la serie RQA, fueran hechos con la caja de herramientas TOCSY Caja de herramientas para sistemas complejos para Matlab ${ }^{\circledR}$, do Potsidencia for Climate Impact Research (PIK) (software de dominio público disponible en <http://tocsy.agnld.uni-potsdam.de> acceso en 27/04 / 2011).

Los análisis de las series temporales no lineales, en lo que se refiere a los aspectos dinámicos de la divergencia de las órbitas de los atractores que es dado por la Expo de Lyapunov $\lambda$, y en cuanto a los aspectos geométricos de los atractores dados por la Dimensión de Correlación D_2, fueron calculados respectivamente por el " programa lyap_r.exe y el programa d2.exe del paquete de software TISEAN - Nonlinear Time Series Analysis.

Las predicciones de las series temporales no lineales se realizaron con el programa zeroth.exe del paquete de software TISEAN - Nonlinear Time Series Analysis.

Los cálculos de los errores de predicción se realizaron utilizando el software libre para uso no comercial IRENE - Integrated Resources for Evaluating Numerical Estimates, que es herramienta de análisis de datos con amplios recursos estadísticos volcados a las técnicas de evaluación de modelos.

\subsubsection{MEDIDAS DE ERROR DE PREDICCIÓN}

La manera de evaluar la distancia entre los valores de las series temporales medidas y los valores de las series temporales predichas es a través de las medidas de los errores de predicción (SAVI, 2006). Para este trabajo se adoptaron cuatro medidas para cuantificar el error en la predicción, siendo ellas: el Coeficiente de Correlación r, el coeficiente de Willmott d, la raíz cuadrada del error medio RMSE y el error medio absoluto MAE, descritas a continuación.

\subsubsection{COEFICIENTE DE CORRELACIÓN R}

La precisión de una predicción es dada por el Coeficiente de Correlación r. Este índice estadístico indica el grado de asociación entre dos magnitudes, en el caso de este trabajo, entre la serie temporal medida (M) y la serie temporal predice $(P)$. El Coeficiente de Correlación $r$ es dado por la ecuación:

$$
r=\frac{\sum_{i=1}^{N}(M-\bar{M})(P-\bar{P})}{\sqrt{\left[\sum_{i=1}^{N}(M-\bar{M})^{2}\right]\left[\sum_{i=1}^{N}(P-\bar{P})^{2}\right]}}
$$

De modo que el grado máximo de asociación es que $r=1$ significa una correlación perfecta positiva entre las dos series temporales, $r=-1$ significa una correlación negativa perfecta entre las dos series, es decir, si una aumenta la otra disminuye,$=0$ que las series no tienen asociación una con la otra. 


\subsubsection{COEFICIENTE DE WILLMOTT D}

La concordancia se refiere a la exactitud o al acercamiento de las series temporales predichas a las medidas. Para cuantificar matemáticamente esta aproximación, fue desarrollado por Willmott (1982) un coeficiente designado concordancia o exactitud $d$. Sus valores varían de $d=0$, para ninguna concordancia, a $d=1$ para la concordancia perfecta. El índice es dado por la siguiente ecuación:

$$
d=1-\left[\frac{\sum_{i=1}^{N}(P-M)^{2}}{\sum_{i=1}^{N}(|P-\bar{M}|+|M-\bar{M}|)^{2}}\right]
$$

Eq. 4

\subsubsection{RAÍZ CUADRADA DEL ERROR MEDIO RMSE}

La raíz cuadrada del error medio RMSE es una de las estadísticas más comunes de medición de errores, definida como:

$$
R M S E=\sqrt{\frac{1}{p} \sum_{n=N+1}^{N+p}\left(M_{n}-P_{n}\right)^{2}}
$$

Eq. 5

\subsubsection{ERROR DE NIVEL ABSOLUTO MAE}

El error medio absoluto MAE es la cantidad utilizada para medir cuán cercanas están los datos previstos de los datos medidos. EI MAE es dado por la ecuación:

$$
M A E=\frac{1}{N} \sum_{i=1}^{N}|P-M|
$$

Eq. 6

\section{RESULTADOS Y DISCUSIÓN}

\subsection{DESCRIPCIÓN MICROCLIMÁTICA}

En esta sección se hace la descripción micrometeorológica del área de estudio en términos de las variables medidas y de la estacionalidad (Tabla 1). El saldo de radiación fue significativamente afectado por las estaciones del año. El saldo de radiación tuvo mayor media \pm desviación estándar durante la estación lluviosa $\left(11,37 \pm 4,06 \mathrm{MJm}^{-2} \mathrm{dia}^{-1}\right)$ que durante la estación seca $\left(7,40 \pm 1,97 \mathrm{MJm}^{-2} \mathrm{dia}^{-1}\right)$. Esta diferencia es esperada debido al factor astronómico, pues la estación lluviosa ocurre en el período de mayor incidencia de radiación solar en el hemisferio sur. 
Tabla 1 - Media mensual y estacional y desviación estándar mensual y estacional del saldo de radiación $(R n)$, de la temperatura del aire $(T)$ y de la humedad relativa $(R h)$.

\begin{tabular}{cccc}
\hline Variables $/ \mathbf{m e s}$ & $\boldsymbol{R} \boldsymbol{n}\left(\boldsymbol{M J M}^{-2} \mathbf{d i a}^{-\mathbf{1}}\right)$ & $\left.\boldsymbol{T}{ }^{\circ} \boldsymbol{C}\right)$ & $\boldsymbol{R} \boldsymbol{h}(\%)$ \\
\hline Enero & $13,10 \pm 3,95$ & $26,57 \pm 1,39$ & $80,79 \pm 8,10$ \\
Febrero & $12,98 \pm 4,22$ & $26,82 \pm 1,32$ & $82,21 \pm 5,51$ \\
Marzo & $12,65 \pm 3,71$ & $27,32 \pm 1,40$ & $81,30 \pm 5,54$ \\
Abril & $10,44 \pm 2,88$ & $26,62 \pm 1,97$ & $79,48 \pm 6,58$ \\
Mayo & $7,85 \pm 2,32$ & $23,64 \pm 3,61$ & $76,45 \pm 7,81$ \\
Junio & $6,65 \pm 1,41$ & $22,92 \pm 3,09$ & $71,69 \pm 9,96$ \\
Julio & $6,85 \pm 1,87$ & $23,42 \pm 4,04$ & $61,44 \pm 12,49$ \\
Agosto & $8,65 \pm 1,88$ & $25,02 \pm 3,66$ & $54,02 \pm 14,24$ \\
Septembre & $9,19 \pm 3,05$ & $26,43 \pm 3,63$ & $57,33 \pm 13,85$ \\
Octubre & $10,88 \pm 4,11$ & $27,08 \pm 2,14$ & $71,67 \pm 10,10$ \\
Noviembre & $12,70 \pm 3,84$ & $27,09 \pm 1,81$ & $75,21 \pm 9,22$ \\
Diciembre & $12,57 \pm 4,15$ & $26,42 \pm 1,35$ & $80,69 \pm 8,39$ \\
\hline Anual & $10,37 \pm 4,04$ & $25,78 \pm 3,07$ & $72,63 \pm 13,73$ \\
\hline Seca & $7,40 \pm 1,97$ & $23,79 \pm 3,73$ & $62,27 \pm 14,35$ \\
Lluvia & $11,37 \pm 4,06$ & $26,79 \pm 2,49$ & $76,13 \pm 11,38$ \\
\hline
\end{tabular}

La temperatura del aire fue significativamente afectada por las estaciones del año. Las medias mensuales de la temperatura \pm desviación estándar fueron mayores durante la estación lluviosa $(26,79 \pm 2,49 \circ \mathrm{C})$ que estación seca $\left(23,79 \pm 3,73^{\circ} \mathrm{C}\right)$. Los mayores valores de las temperaturas medias durante la estación lluviosa están relacionados con la mayor disponibilidad de energía radiante. La reducción en los valores de la temperatura del aire en la estación seca, además de la menor disponibilidad de energía radiante, puede haber sido resultado de la ocurrencia de frentes fríos. En estas situaciones de frentes fríos, ocurren caídas bruscas de la radiación incidente, debido a la presencia de nebulosidad. Durante estos eventos, la temperatura máxima puede ser $10^{\circ} \mathrm{C}$ más baja que la temperatura máxima del día anterior. Además, dependiendo del volumen de aire frío, la temperatura puede tardar de 2 a 3 días para regresar a los valores registrados antes del paso del frente frío (FISCH, 1996, BIUDES et al., 2012).

La humedad relativa del aire fue significativamente afectada por las estaciones del año. Los promedios mensuales de la humedad relativa \pm desviación estándar fueron mayores durante la estación lluviosa $(76,13 \pm$ $11,38 \%)$ que en la estación seca $(62,27 \pm 14,35 \%)$. El aumento de la humedad relativa del aire en la estación lluviosa fue resultado de la mayor presencia de vapor de agua en el aire, asociado a la mayor precipitación (ALVES et al., 1999, BIUDES et al., 2012). Los menores valores de humedad relativa del aire en la estación seca están relacionados con la menor disponibilidad de agua en el suelo, lo que causa disminución en la evapotranspiración y consecuentemente en el flujo de calor latente (WRIGHT et al., 1996; BIUDES et al., 2009; BIUDES et al, 2012).

La Figura 3 ilustra, a partir de medias mensuales de septiembre de 2006 hasta agosto de 2010, el patrón similar de variabilidad estacional de las 
variables. Se destacan las menores medias de Rn en junio y julio, y las mayores de noviembre a marzo. La T también exhibió los menores valores en junio y julio, y los mayores valores de septiembre hasta abril. Por fin la RH exhibió los menores valores en el período más lejano de la estación lluviosa, o sea, julio, agosto y septiembre.

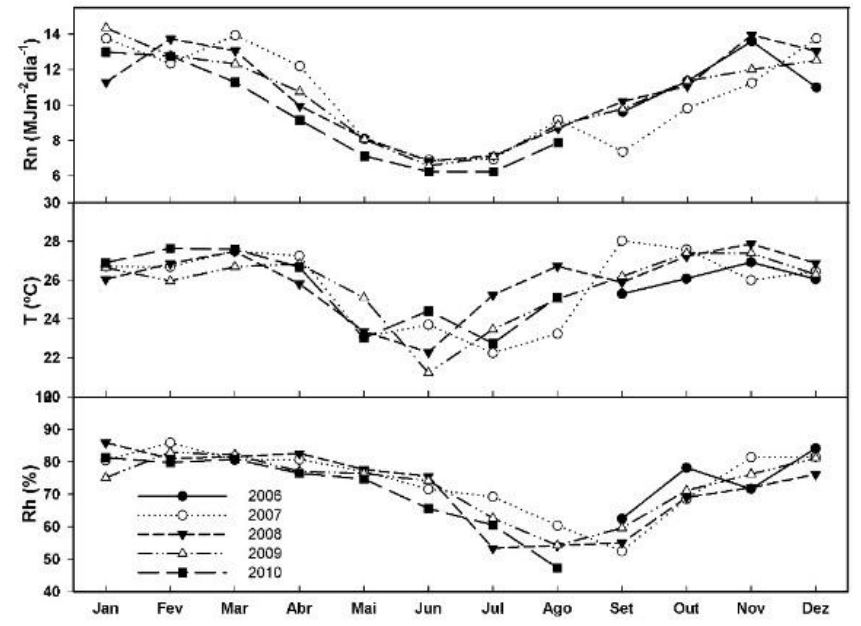

Figura 3 - Media mensual del saldo de radiación (Rn), de la temperatura del aire $(T)$ y de la humedad relativa (Rh).

Los valores mayores de los promedios de la temperatura del aire y humedad relativa en la estación lluviosa también son debidos al efecto liberador / moderador del término de almacenamiento de energía de la biomasa, o sea, durante la noche, la liberación de energía por la biomasa del bosque actúa en el sentido de mantener el balance radioactivo nocturno y no permite enfriamiento acentuado de la superficie (FISCH, 1996; BIUDES et al., 2012).

La precipitación en este período, según Biudes et al. (2013), fue significativamente afectada por las estaciones del año, con menores valores durante la estación seca reflejando el $6 \%$ y mayores valores en la estación lluviosa con el $94 \%$ del total precipitado.

\subsection{ANÁLISIS DE LAS SERIES TEMPORAIS NO LINEALES}

En las siguientes cuatro secciones se presentan los resultados de los análisis de series temporales no lineales haciendo uso de cuatro herramientas de la Teoría de los Sistemas Dinámicos, siendo ellas: Ios Gráficos de Recurrencia RP, el Análisis de Cuantificación de Recurrencia RQA, el Expoente de Lyapunov $\lambda$ y la Dimensión de Correlación D_2.

\subsubsection{GRÁFICOS DE RECURRENCIA RP}

El gráfico de recurrencia RP es una técnica avanzada de análisis de datos no lineales. Es una vista (o un gráfico) de una matriz cuadrada, en la que los elementos de la matriz corresponden a los momentos en que un determinado estado de un sistema dinámico es recurrente. En el caso de que se trate de un sistema de gestión de la calidad, se debe tener en cuenta que, Según Eckmann 
et al. (1987) los RP presentan dos características principales, siendo una de gran escala llamada "tipología" que son los resultados cualitativos presentados en esta Sección, y una de pequeña escala llamada "textura" que son los resultados cuantitativos (la RQA) presentados en la próxima Sección.

El objetivo inicial del RP es la inspección visual de las trayectorias en el espacio de fase de dimensiones superiores $(m>3)$. La visión de un RP provee indicios sobre la evolución temporal de esas trayectorias. Se presentan en la Figuras 4 los RP de los atractores asociados a las series temporales de las variables micrometeorológicas estudiadas.

En estos RPs fueron destacados con una línea la separación entre los años, y en gris los períodos secos de cada año. Para la mejor identificación, los aspectos de la descripción cualitativa de la tipología de los RPs fueron destacados en rojo y numerados.

De la serie temporal microclimática $\mathrm{Rn}$ se generó el 『RP》_Rn (Figura 4a) que presentó una tipología que osciló estacionalmente entre periódica y aleatoria. La inspección visual del $\llbracket R P \rrbracket \_R n$ posibilita dos interpretaciones. Por un lado, se observa una recurrencia periódica atribuida a las agrupaciones (clusters) en forma de ajedrez y también a los espacios en blanco indicadores de que la evolución de los estados del sistema fue semejante en momentos diferentes, y aunque algunos estados no cambian o cambian muy lentamente durante el tiempo, indicando comportamiento laminar. Por otro lado, las observaciones de puntos aislados, que son representativos de estados raros, de fuerte fluctuación del sistema, o que no persisten por mucho tiempo indican un comportamiento aleatorio.

La visualización del 『RP』_Rn como un todo posibilita identificar la duración de las agrupaciones en forma de ajedrez, que ocurrieron en los tres meses del período seco en todos los años analizados, respectivamente en los meses de junio, julio y agosto. Los meses de marzo, abril y mayo, período en que el Pantanal se encuentra inundado, localizado temporalmente en la separación entre las agrupaciones y los estados aleatorios, presentó estructuras de líneas rectas significando que los estados están cambiando lentamente y también líneas curvas y largas significando que la dinámica del sistema está cambiando. Las regiones casi sin puntos son indicadoras de prácticamente ninguna recurrencia, y ocurrieron en la intersección de las dos tipologías, o sea, los meses junio, julio y agosto del período seco poseen una recurrencia prácticamente nula en relación a los demás meses del año, evidenciando la diferencia estacional del comportamiento dinámico del Rn. 


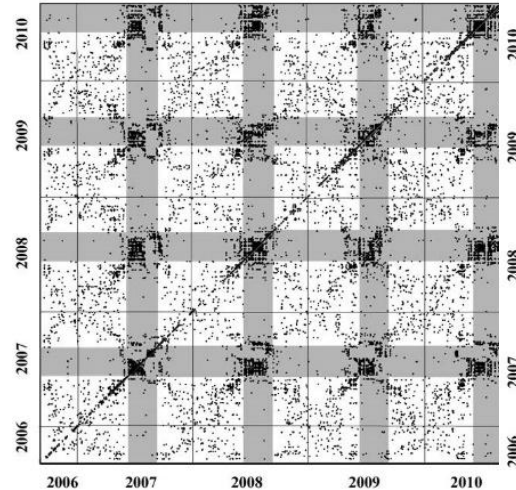

(a)

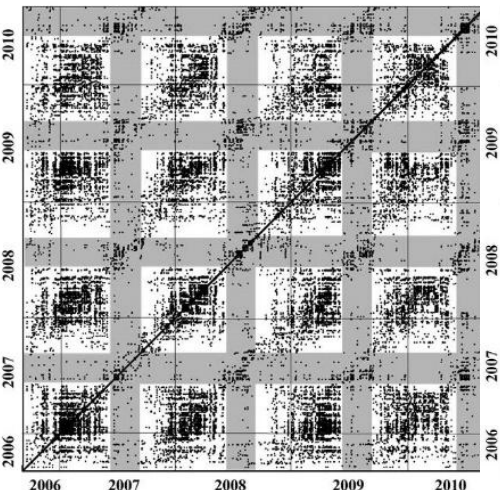

(b)

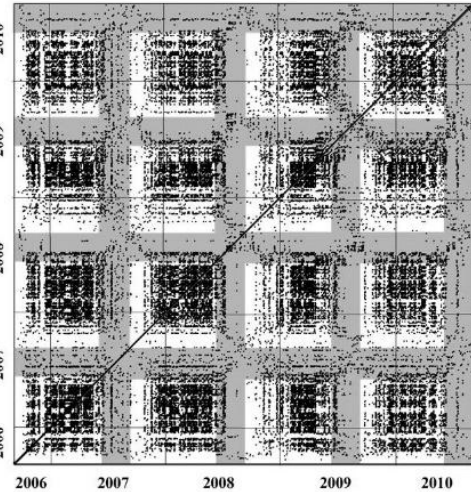

(c)

Figura 4 - (a) Gráficos de Recurrencia RP de la variable microclimática Rn; (b) Gráficos de Recurrencia RP de la variable microclimática T; y (c) Gráficos de Recurrencia RP de la variable microclimática $\mathrm{Rh}$.

La serie temporal microclimática $T$ fue utilizada para generar el $\llbracket R P \rrbracket-T$ que presentó una tipología estándar de sistemas casi-periódicos con transiciones de fase (Figura 4b). En la inspección visual del 『RP】_T se observa una recurrencia casi periódica atribuida por poseer agrupamientos (clusters) en forma de ajedrez en los dos períodos, el seco y el lluvioso. En los períodos secos las agrupaciones son menores y en los períodos lluviosos las agrupaciones son mayores. En cuanto a estas recurrencias de estados (agrupamientos), no se producen en la intersección entre los períodos secos y lluviosos del 『RP】_T, de modo que los espacios en blanco llenan predominantemente estas regiones junto con algunos puntos de estados raros. La interrupción entre los dos grupos, los secos y los lluviosos, se rellenan con estructuras de líneas rectas significando que los estados están cambiando muy lentamente y también con líneas curvas y largas que significan la dinámica del sistema está cambiando. De ello se desprende que la estacionalidad de la dinámica de la $T$, visto las diferentes recurrencias características en cada período del año, el seco y el lluvioso, debe ser considerada en la previsión de series temporales.

El 【RP』_Rh resultado de la serie temporal Rh presentó una tipología estándar de sistemas casi-periódicos con transiciones de fase (Figura 4c). La inspección visual del \RP》_Rh permite observar una recurrencia casi periódica poseedora de grandes agrupaciones (clusters) en forma de ajedrez que se inician en el segundo mes del período lluvioso, en el caso del mes de noviembre, y perduran hasta el primer mes del período en el mes de junio. Entre estas agrupaciones del $\llbracket R P \rrbracket \_$Rh tienen desfase de uno a dos meses con relación a los períodos secos y lluviosos. La transición entre las agrupaciones y los espacios en blanco se rellenan con estructuras de líneas rectas que significan que los estados están cambiando muy lentamente. Los espacios en blanco con algunos puntos raros en los meses de julio, agosto, septiembre y octubre posibilitan indicar que $\mathrm{Rh}$ posee una dinámica con estados que no se repiten o ocurren muy poco haciendo la previsión micrometeorológica un problema de difícil solución.

Los Gráficos de Recurrencia RP son reconocidos como una herramienta poderosa para el análisis de datos, no sólo para la visualización de las estructuras de la serie temporal, sino también para estimar sus invariantes topológicas. A través del RP una gran cantidad de información es codificada en una representación bidimensional mostrando que bajo algunas condiciones es 
posible reconstruir un atraco a partir del RP, por lo menos, topológicamente. Esto significa que toda la información relevante de la dinámica está contenida en la textura (THIEL et al., 2004).

Los resultados de la textura de los RP a través del Análisis de Cuantificación de Recurrencia RQA se presentarán en la próxima Sección.

\subsubsection{ANÁLISIS DE CUANTIFICACIÓN DE RECURRENCIA - RQA}

El análisis de cuantificación de recurrencia RQA transforma las representaciones gráficas del RP en estadísticas (ZBILUT \& WEBBER Jr., 1992; WEBBER Jr \& ZBILUT, 1994; MARWAN et al., 2002). Se presentan a continuación los resultados de cuatro Análisis Cuantitativos de Recurrencia RQA, siendo la Razón de Recurrencia RR, el determinismo DET, el Longitud Medio de la Diagonal L y la Entropía ENTR.

Los parámetros de desfase temporal $\mathrm{T}$ y de tamaño de inmersión $m$ utilizados para cada una de las RQA de los RP son los mismos presentados en la Sección 3.3. En cuanto al radio de la vecindad $\varepsilon$ (es decir, el umbral de sondeo) se utilizó el valor del $10 \%$ del promedio de la serie temporal, que corresponde aproximadamente a la desviación estándar de la serie de datos (MINDLIN \& GILMORE, 1992; KOEBBE \& KRESS, 1992; ZBILUT \& WEBBER, 1992; ZBILUT et al., 2002).

Debido al número elevado de datos de las series temporales analizadas y sus respectivas limitaciones computacionales, se optó en hacer la RQA de los RP a través de sucesivas ventanas móviles (Windows shift) con pasos fijos hasta cubrir toda la serie temporal. Las series temporales teóricas tuvieron una ventana móvil midiendo el $10 \%$ del tamaño de la serie y un paso midiendo el $1 \%$ del tamaño de la serie. Las series temporales micrometeorológicas, que poseen 140 mil datos, fueron analizadas con una ventana móvil midiendo 3 mil, datos que corresponden a aproximadamente un mes, y con un paso de 96 datos que corresponde a un día, totalizando así 1430 análisis para cada una de las seis medidas de RQA utilizadas en este trabajo. La utilización de ventanas móviles permitió detectar las variaciones sensibles de la dinámica del sistema micrometeorológico durante el período de los cuatro años de datos medidos.

A priori, en las figuras de las tres series temporales micrometeorológicas (con los datos estandarizados) se observa un patrón estacional bien comportado, lo que también puede ser observado en la figura de la varianza. Es importante notar que incluso la varianza se ha presentado flotando alrededor del valor 1 durante los 4 años de las series, los años no fueron iguales.

La densidad de puntos de recurrencia medida 『RR』_Rn presentó valores menores que $1 \%$ oscilando entre $1 \times 10-3$ y $2 \times 10-3$ en el período lluvioso a $5 \times 10-3$ y $8 \times 10-3$ en el período seco. No hubo pico en la sequedad de 2009 . La

【RR】_T osciló estacionalmente con mayor densidad de recurrencia en los períodos lluviosos, alrededor de $3 \times 10-4$ y un gran pico aislado en la transición lluvia seca de 2009 alcanzando 5x10-4, y valles menores de 0,1×10-4 en la sequía con un pico aislado $(2 \times 10-4)$ en la sequía de 2010 . En cuanto a 【RR】 _Rh, los valores encontrados, aunque con fluctuaciones, denota una mayor recurrencia en el período lluvioso ( $1 \times 10-4$ hasta $2 \times 10-4)$ que en el período seco $(0,5 \times 10-4)$. Se presta especial atención a un pico aislado midiendo 0,5x10-4 en la transición Iluvia-seca de 2009. El comportamiento identificado en la transición 


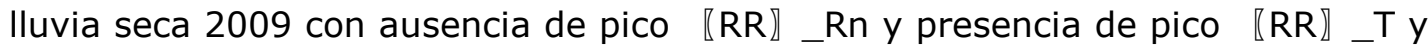
【RR】_Rh puede estar relacionado con el fin de la inundación del Pantanal. En este período el suelo Pantaneiro aún se mantiene empapado resultando en altos valores de evaporación del suelo lo que estabiliza la humedad y también la temperatura.

La predicción del sistema es dada por la DET que varía de 0 (cero por ciento) a ninguna previsión hasta 1 (ciento por ciento) para una óptima previsión. La medida 『DET』_Rn osciló estacionalmente con picos bien definidos de $80 \%$ en el período seco y $10 \%$ en el valle del período lluvioso, que a su vez va acompañado de un pequeño pico aislado en el medio de ese mismo período con amplitud en torno al 40\%. El 『DET》_T presentó fluctuación en torno al $60 \%$ en todo el análisis, acompañado de valles en el período seco en torno al $20 \%$. Se observa la ausencia de estos valles en el período seco de 2009 . En cuanto a la 『DET》_Rh, ésta presentó fluctuaciones en torno del $40 \%$ en todo el análisis con algunos valles midiendo el $10 \%$ y picos midiendo los $70 \%$ localizados en las transiciones entre las estacionalidades.

La medida de la longitud media de la diagonal L (también llamada medida de complejidad) representa el tiempo promedio en que dos segmentos de la trayectoria del atractor "caminan" cerca una de la otra. Se estableció como longitud mínima I como siendo de 2 estados en el RP, luego los datos de L trazados se refieren a longitudes I de dos estados. Así el L_Rn presentó comportamiento estacional con picos mayores en el período seco midiendo 8 estados del atractor, y valles el período lluvioso midiendo 4 estados acompañados de pequeños picos menores en esos valles midiendo cerca de 6 estados. El L_T presentó fluctuaciones en torno a 6 estados con algunos picos en la sequía llegando a 10 estados. La L_Rh presentó fluctuaciones en torno a 5 estados con un pico de 12 estados en la sequía de 2009.

La frecuencia de distribución de las líneas diagonal, medida ENTR, se da como la suma de todas las longitudes recurrentes escalonadas en la escala logarítmica. Su interpretación es que cuanto mayor sea el valor de la entropía, más organizado está el sistema, mayor es su complejidad y los estados tienden a ser más parecidos o iguales. Por otro lado, menor que los valores de la entropía, el sistema será menos complejo, sus datos más aleatorios y los estados (los datos) más diferentes. Así, la 『『ENTR】_Rn presentó mayores distribuciones de frecuencias en los períodos secos con picos cercanos al valor 2 , decreciendo en el período lluvioso con valores cada vez menores llegando a cero en la lluvia 2008-2009 y 2009-2010. La 『『ENTR』_T presentó fluctuaciones alrededor del valor 1 y 1,5 durante todo el análisis. Obtuvo en el período seco tres picos cercanos al valor 2 y dos valores cero (0). También se registraron tres valles con valores 0,5 en el período Iluvioso. Esto demuestra que la complejidad del sistema no es necesariamente dependiente de la estacionalidad. La medida 『ENTR】_Rh se comportó con fluctuaciones entre los valores 0,5 y 1 en todo el análisis, con cinco valles llegando hasta cero y siendo tres de ellos próximos al final del período seco. 

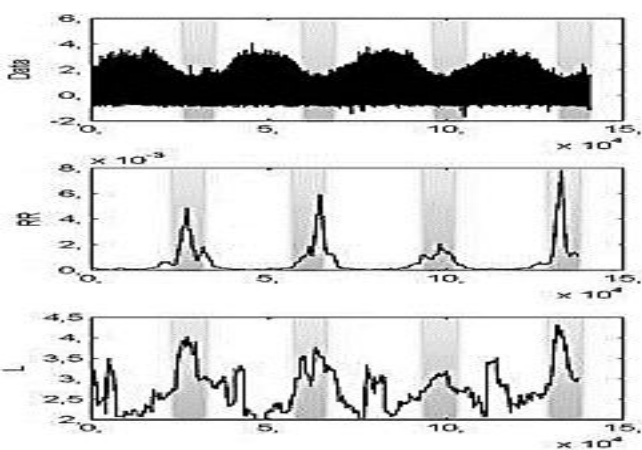
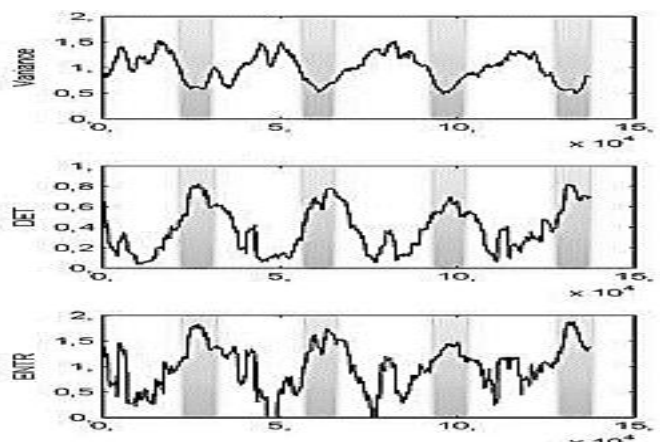

Figura 5 - Análisis de Cuantificación de Recurrencia RQA de la variable microclimática Rn.
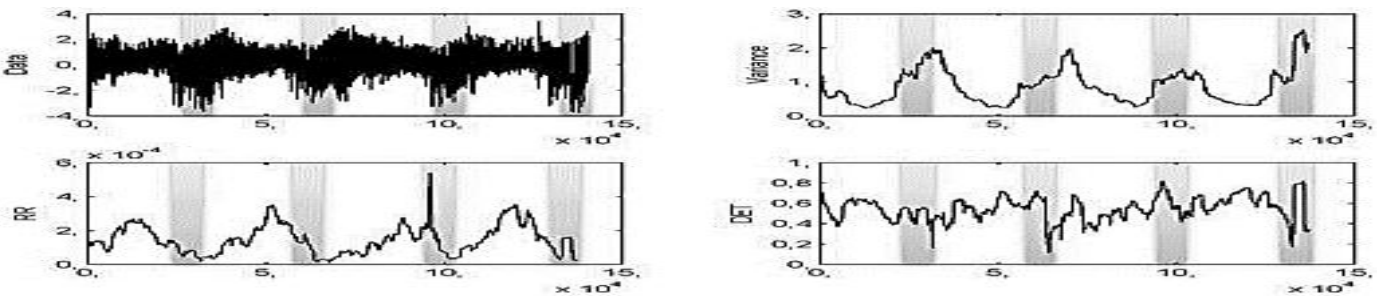

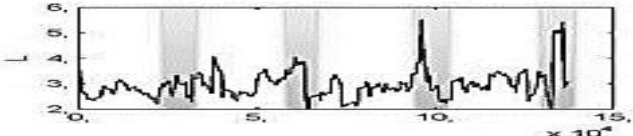

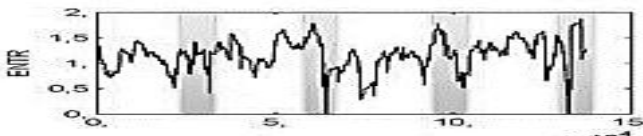

Figura 6 - Análisis de Cuantificación de Recurrencia RQA de la variable microclimática T.
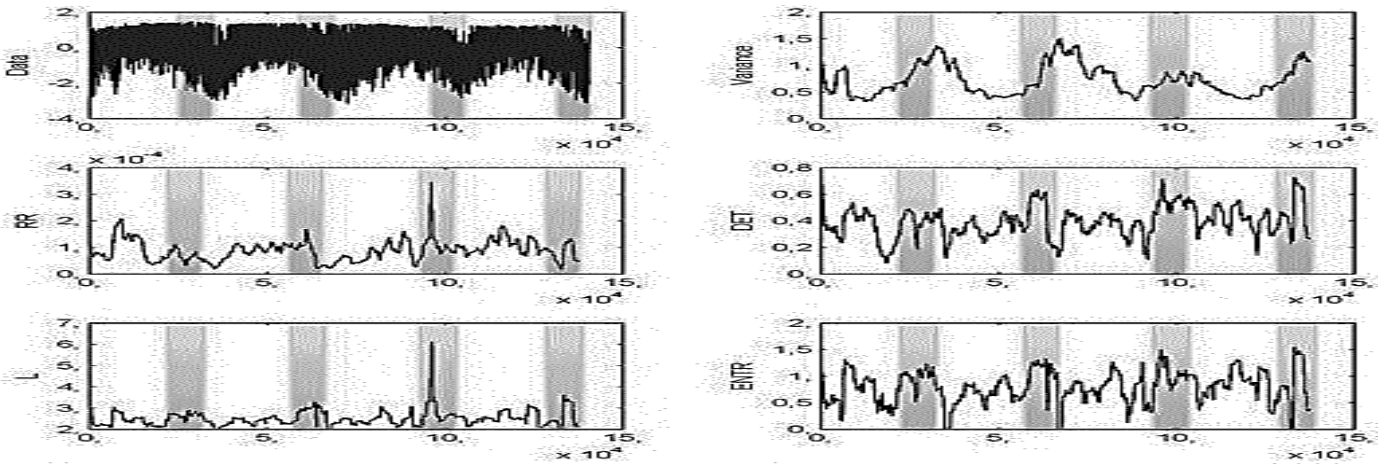

Figura 7 - Análisis de Cuantificación de Recurrencia RQA de la variable microclimática Rh.

\subsection{PREDICCIÓN NO LINEAL Y VERIFICACIÓN DEL MODELO}

El método de la predicción no lineal simple utilizado en este trabajo posibilita hacer predicciones sin que se conozcan las ecuaciones de gobierno del fenómeno físico asociado al sistema (SAVI, 2006). Este método se basa en la reconstrucción del atractivo asociado a una serie temporal medida, partiendo de una serie temporal escalar (pasado) con el objetivo de determinar una estimación para sus valores siguientes (futuro) proporcionando información precisa sobre la dinámica del sistema (KANTZ \& SCHREIBER, 2004). Así se hacen previsiones sin la construcción explícita de un modelo para una serie temporal específica. 
A continuación se presentan los resultados estimados de los parámetros que involucran la predicción, tales como el tiempo máximo de predicción fiable $\xi$ basado en el inverso del exponente de Lyapunov $\lambda$, la predicción no lineal simple comparada con las series temporales medidas junto con las medidas de errores de la predicción.

\subsubsection{TIEMPO MÁXIMO DE PREVISIÓN DE CONFIANZA}

Se muestra en la Tabla 2 el tiempo máximo de predicción fiable $\xi$ (también llamado "tiempo de Lyapunov"), basado en la idea de que si el atraco posee al menos un $\lambda_{\max }>0$, entonces el crecimiento de las perturbaciones es controlado por $\lambda_{\max }$ que conduce a una divergencia exponencial de las trayectorias. Por lo tanto, la manera cuantitativa de estimar la previsibilidad del sistema, llamado tiempo máximo de predicción fiable $\xi$, se define como el intervalo de la muestra experimental $\mathrm{T}$ dividido por el mayor exponente de Lyapunov $\lambda_{\max }$ (TSONIS, 2001; GOMES \& VARRIALE, 2004; CHAUDHURI, 2006; MILLÁN et al., 2009).

Tabla 2 - Tiempo máximo de predicción confiable $\xi$ (tiempo de Lyapunov).

\begin{tabular}{|c|c|c|c|c|}
\hline Variables/Parámetros & $\lambda_{\text {global }}$ & $\xi_{\text {global }}$ & $\lambda_{\text {local }}$ & $\xi_{\text {local }}$ \\
\hline$R n$ & 0,0091 & $\begin{array}{l}109 \text { dados } \\
\text { ou } 1,14 \text { dias }\end{array}$ & 0,0030 & $\begin{array}{l}333 \text { dados } \\
\text { ou } 3,47 \text { dias }\end{array}$ \\
\hline$T$ & 0,0061 & $\begin{array}{l}163 \text { dados } \\
\text { ou } 1,70 \text { dias }\end{array}$ & 0,0020 & $\begin{array}{l}500 \text { dados } \\
\text { ou } 5,21 \text { dias }\end{array}$ \\
\hline$R h$ & 0,0073 & $\begin{array}{l}136 \text { dados } \\
\text { ou } 1,42 \text { dias }\end{array}$ & 0,0025 & $\begin{array}{l}400 \text { dados } \\
\text { ou } 4,16 \text { dias }\end{array}$ \\
\hline
\end{tabular}

Los resultados presentados en la Tabla 2 se presentan en términos del parámetro Mayor Expo de Lyapunov que se calcula para la serie total, es decir, del 1 de septiembre de 2006 hasta el 1 de septiembre de 2010, y que fue llamado de $\lambda_{\text {global }}$, que a su vez generó el parámetro $\xi_{\text {global }}$. De la misma forma, para el parámetro Mayor Expoente de Lyapunov calculado para recortes mensuales de esa misma serie temporal, Ilamado $\lambda_{\text {local }}$ que generó $\xi_{\text {local }}$. Esto se justifica por la fuerte estacionalidad encontrada en el análisis no lineal $R P$ y su cuantificación RQA, indicando la necesidad de la estimación de esos parámetros en términos globales y locales, o sea, no es posible prever series temporales de períodos lluviosos usando datos de sequía, y viceversa.

En la siguiente Sección se presentaron las predicciones de las series temporales utilizando el límite de previsión $\xi$ en términos globales y locales, y sus respectivas medidas de errores de predicción.

\subsubsection{PREDICCIÓN NO LINEAL SIMPLE Y MEDIDA DE ERROR DE PREDICCIÓN}

En esta sección se presentaron los resultados de la predicción no lineal simple de las series temporales de $\mathrm{Rn}, \mathrm{T}$ y $\mathrm{Rh}$ a partir de medidas en torre micrometeorológica en el Pantanal de Mato Grosso. La predicción se hace mes a mes para los doce meses del año 2007 teniendo en cuenta el parámetro tiempo de predicción fiable $\xi$ global y local. Esta postura parte de la evaluación del número de puntos utilizados en la predicción, que después de algunas pruebas, resultó en predicciones mejores cuando se utilizó sólo el mes inmediatamente anterior a los datos a ser predichos. Las series temporales medidas no se 
sometieron a ningún tipo de filtración con el fin de trabajar con la naturaleza intrínseca del sistema micrometeorológico al que se asocia la dinámica de la serie y de evitar perder cualquier información en procesos de filtración.

Por lo tanto, se utiliza una serie medida de un mes anterior (aproximadamente 3000 datos) para estimar una serie prevista referente al mes siguiente, de la cual se miden los errores dentro del tiempo establecido por el parámetro de predicción fiable $\xi$, especificados en la Tabla 2.

Con el objetivo de establecer una verificación del modelo, los resultados de la predicción se trazan junto con los respectivos fragmentos de las series medidas del futuro que se conocen. En seguida se calcularon las medidas de error de predicción comparando la serie predice con la serie medida. De esta forma, se presenta a continuación, mes a mes para los doce meses del año 2007, y para las tres variables, iniciando en la Figura 35 hasta la Figura 70 los resultados de la predicción y sus respectivas medidas de error. Estos resultados, referentes a la verificación del modelo utilizado, demuestran su capacidad de capturar el comportamiento dinámico de las trayectorias de los atratores reconstruidos en el espacio de fase, y consecuentemente el comportamiento general de las tres series temporales micrometeorológicas medidas Rn, T y Rh.

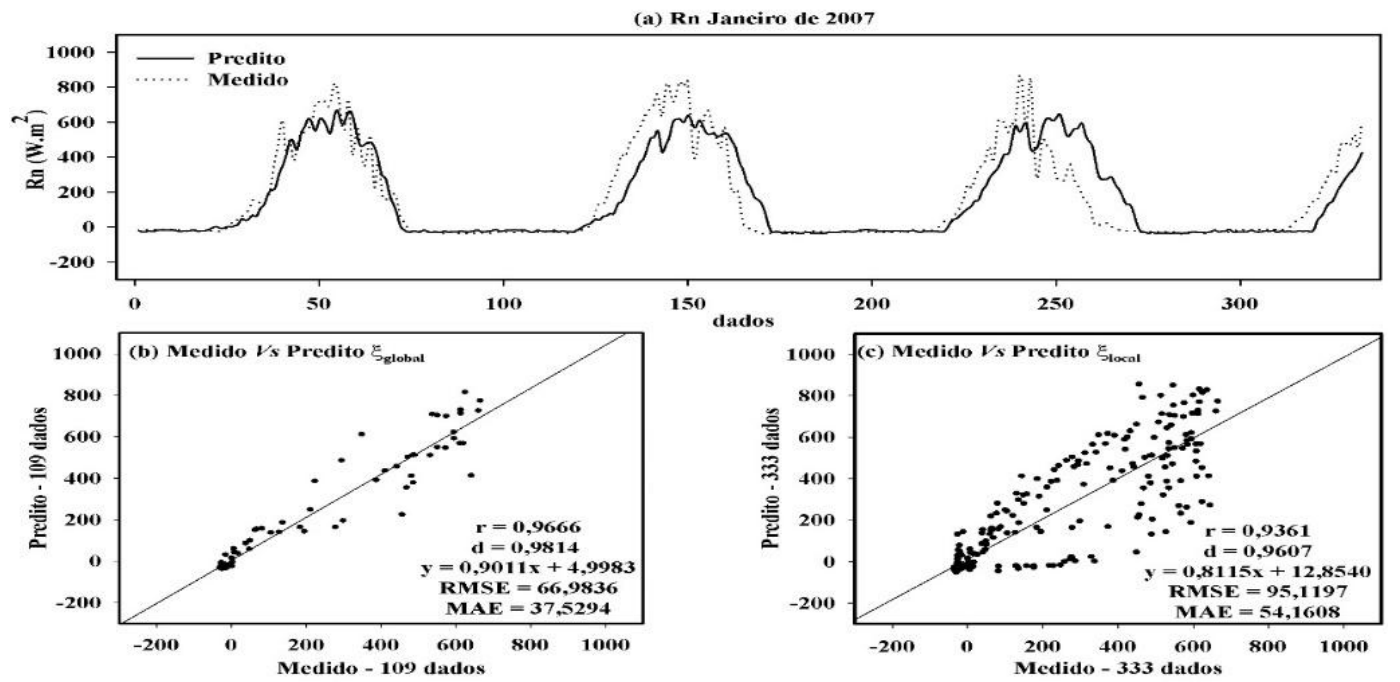

Figura 8 - (a) Serie temporal de Rn medida y predice, enero de 2007. Análisis de errores

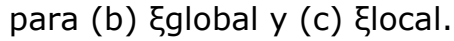




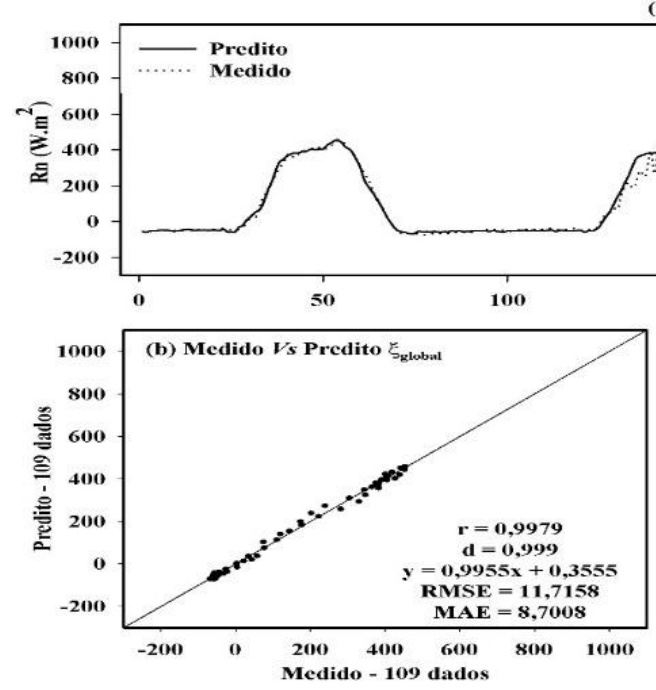

(a) Rn Julho de 2007

Figura 9 - (a) Serie temporal de Rn medida y predice, julio de 2007. Análisis de errores

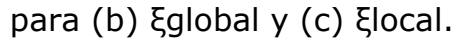
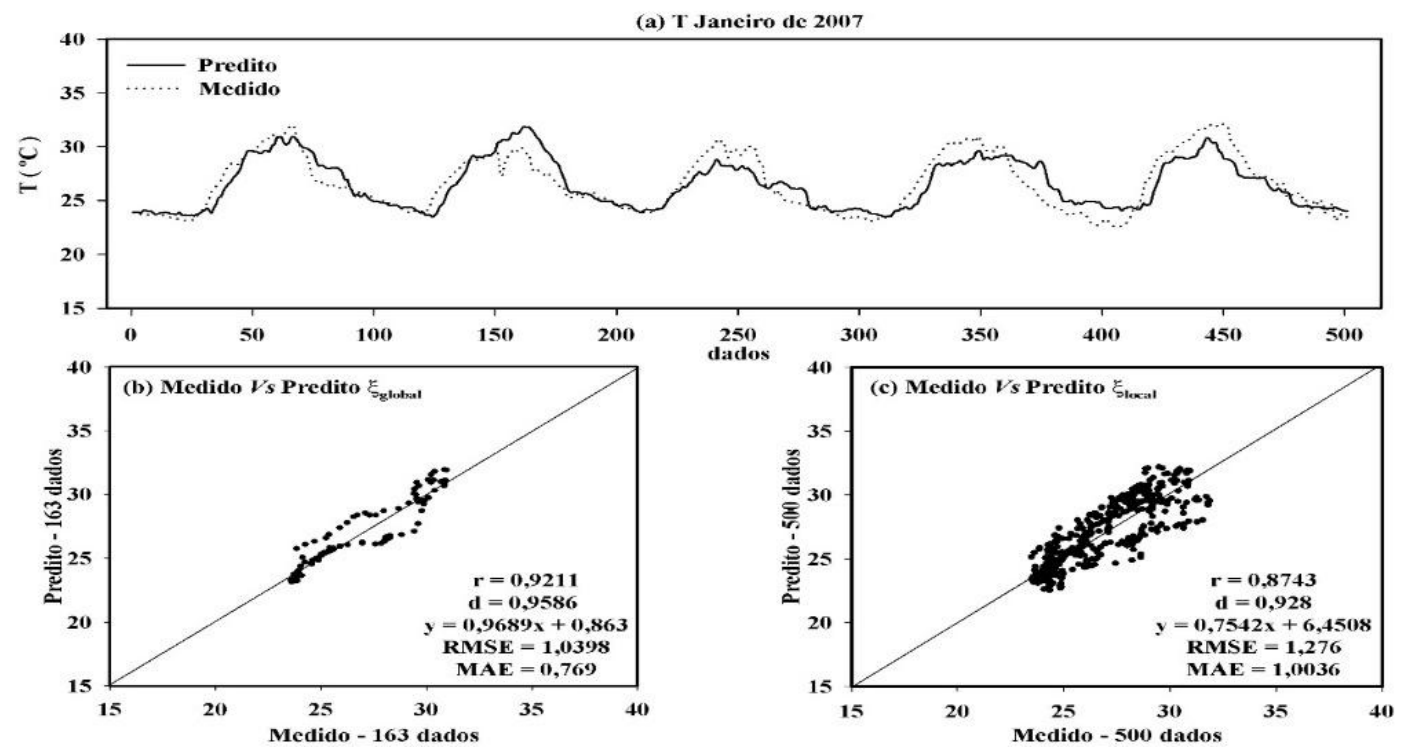

Figura 10 - (a) Serie temporal de T medida y predice, enero de 2007. Análisis de errores

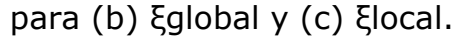



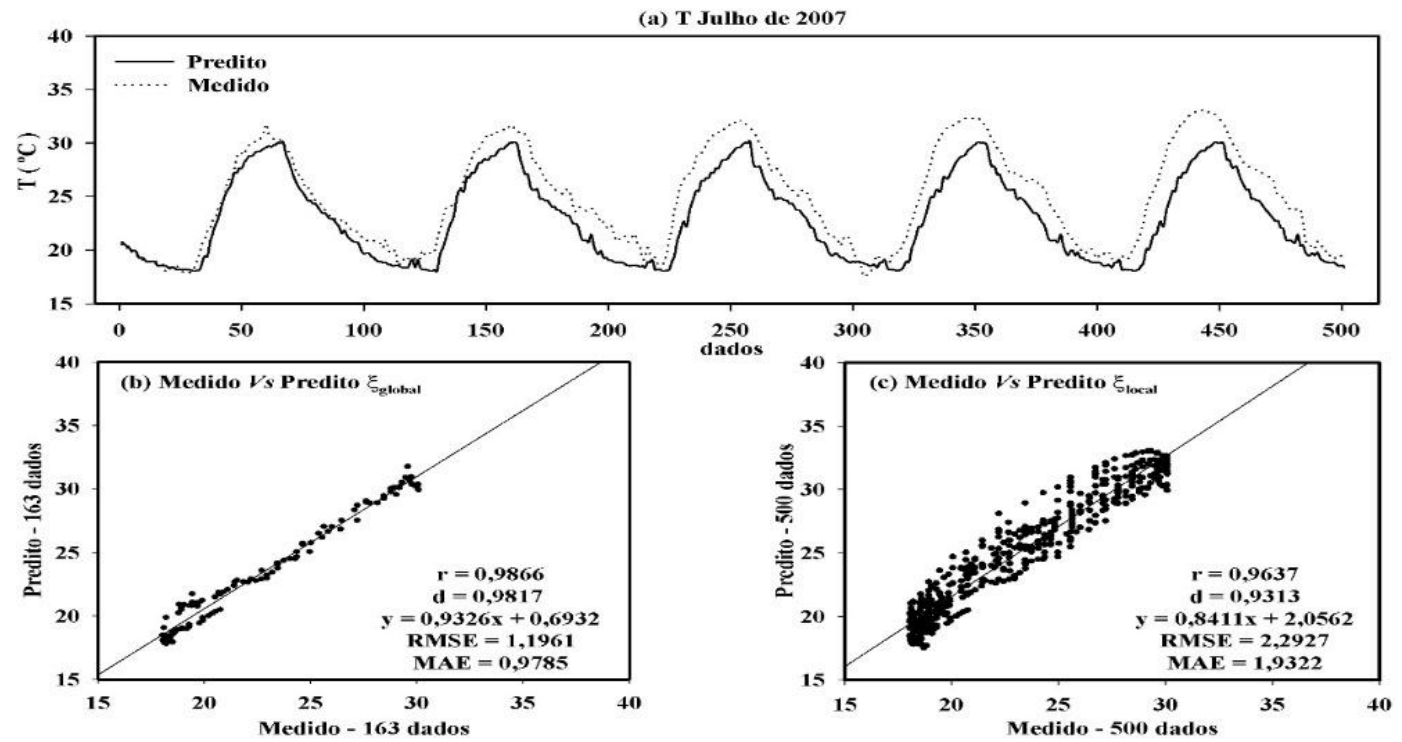

Figura 11 - (a) Serie temporal de T medida y predice, julio de 2007. Análisis de errores

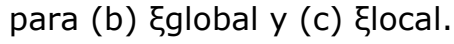

(a) Rh Janeiro de 2007
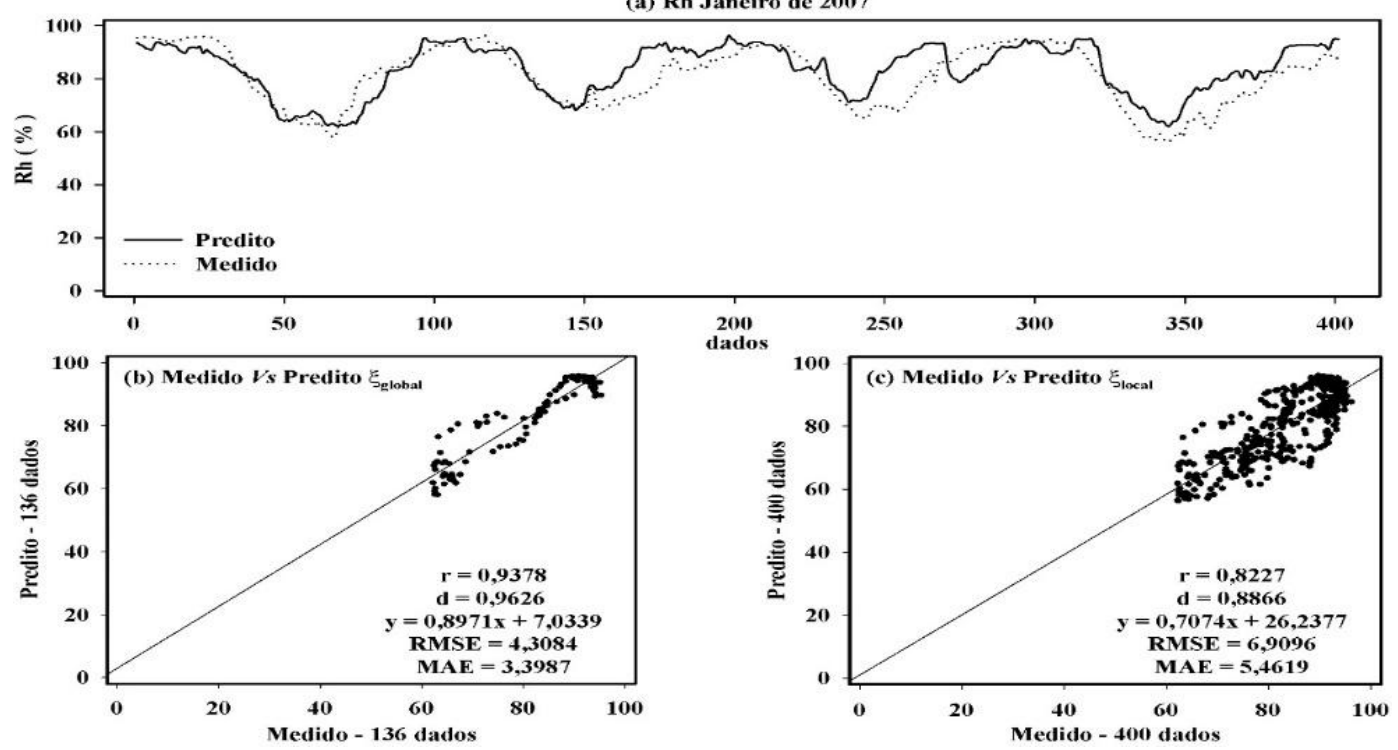

Figura 12 - (a) Serie temporal de Rh medida y predice, enero de 2007. Análisis de

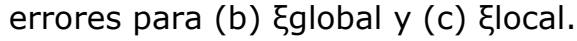



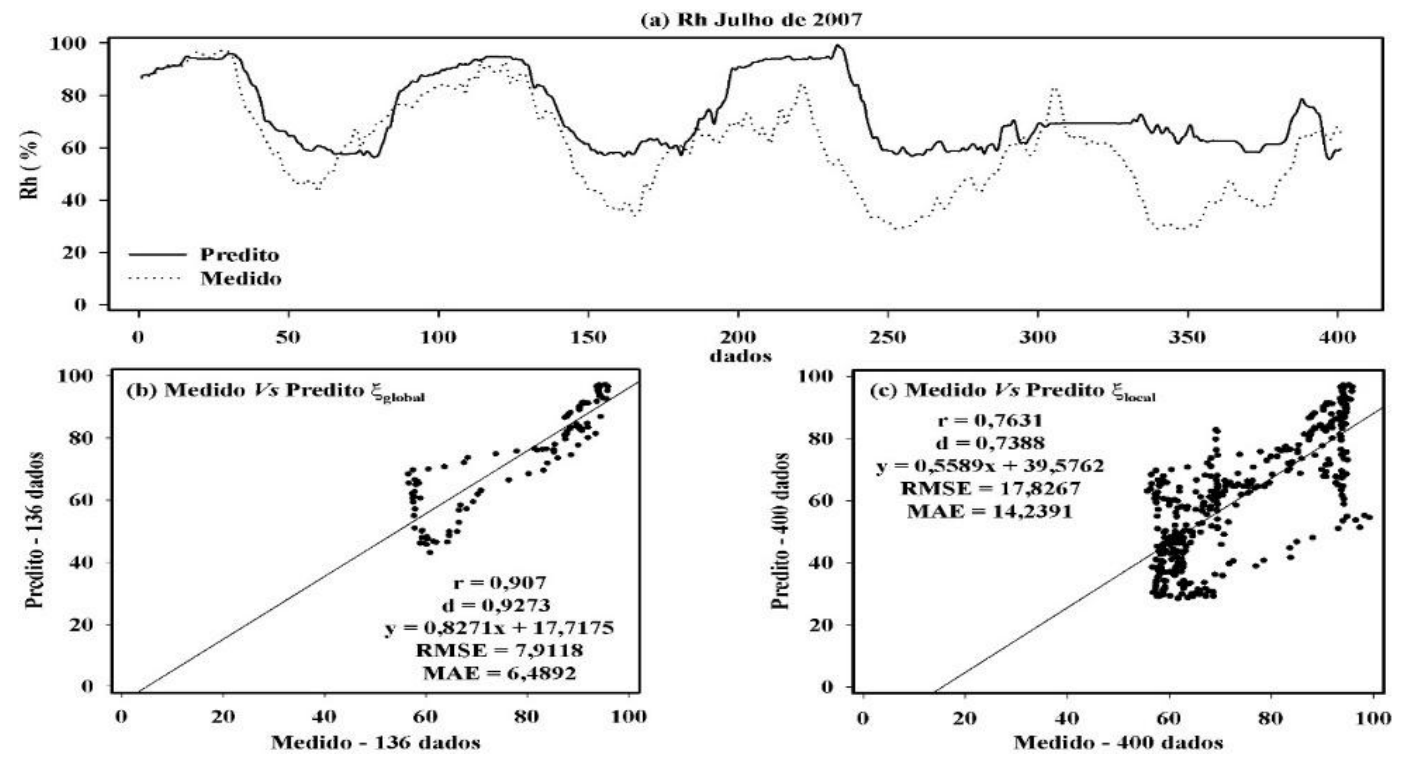

Figura 13 - (a) Serie temporal de Rh medida y predice, julio de 2007. Análisis de errores

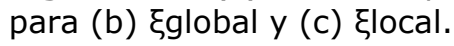

En la Tabla 3 a continuación se presentan los resultados de las medidas de error de previsión en términos de promedios de los períodos seco y lluvioso, y en cuanto al parámetro $\xi$ en global y local.

Tabla 3 - Media y desviación estándar de las medidas de error de predicción de las series temporales micrometeorológicas en cuanto al parámetro $\xi$ y la estacionalidad.

\begin{tabular}{ccccc}
\hline Medidas de Erro & \multicolumn{3}{c}{$\xi_{\text {global }}$} & $\xi_{\text {local }}$ \\
\hline & seca (média) & chuva (média) & seca (média) & chuva (média) \\
\cline { 2 - 5 }$r_{R n}$ & $0,99 \pm 0,01$ & $0,95 \pm 0,03$ & $0,98 \pm 0,01$ & $0,91 \pm 0,01$ \\
$d_{R n}$ & $0,99 \pm 0,01$ & $0,96 \pm 0,02$ & $0,99 \pm 0,01$ & $0,94 \pm 0,05$ \\
$R M S E_{R n}$ & $23,44 \pm 10,29$ & $77,07 \pm 30,74$ & $35,74 \pm 14,38$ & $86,94 \pm 34,78$ \\
$M A E_{R n}$ & $16,81 \pm 7,89$ & $43,26 \pm 17,11$ & $23,08 \pm 8,93$ & $56,32 \pm 24,75$ \\
\hline$r_{T}$ & $0,92 \pm 0,09$ & $0,85 \pm 0,13$ & $0,80 \pm 0,14$ & $0,68 \pm 0,39$ \\
$d_{T}$ & $0,94 \pm 0,04$ & $0,86 \pm 0,11$ & $0,83 \pm 0,11$ & $0,76 \pm 0,22$ \\
$R M S E_{T}$ & $1,85 \pm 0,54$ & $2,18 \pm 0,68$ & $3,93 \pm 1,97$ & $4,51 \pm 5,43$ \\
$M A E_{T}$ & $1,44 \pm 0,41$ & $1,71 \pm 0,53$ & $3,17 \pm 1,49$ & $3,73 \pm 4,72$ \\
\hline$r_{R h}$ & $0,79 \pm 0,08$ & $0,71 \pm 0,25$ & $0,63 \pm 0,12$ & $0,51 \pm 0,25$ \\
$d_{R h}$ & $0,85 \pm 0,07$ & $0,77 \pm 0,17$ & $0,71 \pm 0,02$ & $0,66 \pm 0,14$ \\
$R M S E_{R h}$ & $11,42 \pm 3,85$ & $11,03 \pm 4,69$ & $16,07 \pm 2,84$ & $15,09 \pm 5,12$ \\
$M A E_{R h}$ & $8,87 \pm 2,82$ & $9,03 \pm 3,99$ & $12,78 \pm 2,35$ & $12,01 \pm 4,29$ \\
\hline
\end{tabular}

Las series temporales predichas versus medida de la variable $\mathrm{Rn}$ obtuvieron los mejores resultados de predicción. Los valores de r_Rn y d_Rn, para छglobal y छlocal se mantuvieron cerca de 1 en la sequía y por encima de 0,91 en la lluvia ambos, con errores en torno al 5\% en la sequía y menores que el $10 \%$ en la lluvia. El resultado de $\mathrm{Rn}$ es muy importante ya que es una 
variable directamente relacionada con la entrada y salida de energía del sistema, implicando en ese saldo una conexión directa con el calentamiento o enfriamiento de ese sistema. El mes de marzo presentó los peores resultados de Medidas de Errores de Predicción visto el mes anterior (febrero) que se utiliza para hacer la predicción presentar altos índices de lluvias y nebulosidad.

Las series temporales predichas de la variable $\mathrm{T}$ obtuvieron resultados

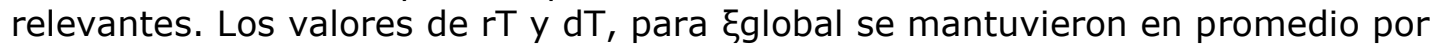
encima de 0,92 en la sequía y por encima de 0,85 en la lluvia; y para छlocal los valores fueron rT y dT por encima de 0,80 en la sequía y por encima de 0,68 en la lluvia. Se resalta también para esta serie temporal, valores pésimos de previsión para el mes de marzo, que por cierto pesaron para bajar la media de los demás parámetros. Excluyendo el mes de marzo, que obtuvo pésima previsión, el promedio de rT y dT para छlocal en la lluvia quedaría por encima de 0,82 . La temperatura es una variable muy importante en el estudio ambiental, ya que es componente de varias otras variables meteorológicas, y una buena previsión de estas series indica que otras más pueden ser bien modeladas. Lo que se suma al hecho de ser fácilmente medida en numerosas estaciones meteorológicas.

En cuanto a las series temporales predichas de la variable Rh obtuvieron resultados medianos. Los valores de $\mathrm{rRh}$ y $\mathrm{dRh}$, para $\xi$ global se mantuvieron cerca de 0,80 en la sequía y por encima de 0,71 en la lluvia, y para छlocal por encima de 0,63 en la sequía y 0,51 en la lluvia. Las predicciones de Rh fueron las que presentaron las menores correlaciones y exacciones y los mayores errores. Esta variable posee una dinámica sensible a la variación de otras variables, principalmente las vinculadas a la circulación atmosférica global. Así como $\mathrm{Rn}$, el mes de marzo para Rh presentó también para esta variable las peores predicciones.

\section{CONCLUSIÓN}

Con el fin de extraer informaciones para la predicción, se hizo la caracterización de las variables según el análisis de series temporales no lineales, en términos cualitativos por Gráficos de Recurrencia identificando en la estacionalidad los períodos cíclicos y los no lineales. En términos cuantitativos, el Análisis Cuantitativo de Recurrencia, en sus medidas de Razón de Recurrencia, Determinismo, Laminaridad y Entropia, proporcionaron la localización en el tiempo y la estimación de esos valores en la serie temporal esos períodos. Se observó una inversión de fase entre el saldo de radiación (mayores picos en el período seco) y la temperatura (mayores picos en el período lluvioso). La humedad relativa presentó tendencia de comportamiento errático.

Por último se aplica el método de predicción no lineal simple cada mes para los doce meses del año 2007, de modo que se utilizó una serie medida de un mes anterior para estimar una serie prevista para el mes siguiente, de la que se verifican la correlación, la exactitud y los errores dentro del tiempo establecido por el parámetro de predicción de confianza.

Los resultados permiten afirmar que el método de predicción no lineal simple capturó la dinámica general de las series temporales micrometeorológicas medidas en el Pantanal Matogrossense. Las series temporales predichas son muy próximas a las series temporales medidas, 
siendo la más cercana el saldo de radiación, luego la temperatura y por fin la humedad relativa del aire. Las diferencias entre las series medidas y las predichas en términos de correlación, exactitud y errores de predicción aumentan en el período lluvioso, y también aumentan en la proporción que se aumenta el tiempo futuro predicho.

Las series temporales predichas versus medida de la variable $\mathrm{Rn}$ obtuvieron los mejores resultados de predicción. Los valores de rRn y dRn, para छglobal y छlocal se mantuvieron cerca de 1 en la sequía y por encima de 0,91 en la lluvia ambos, con errores en torno al $5 \%$ en la sequía y menores que el $10 \%$ en la lluvia. El resultado de $\mathrm{Rn}$ es muy importante ya que es una variable directamente relacionada con la entrada y salida de energía del sistema, implicando en ese saldo una conexión directa con el calentamiento o enfriamiento de ese sistema. El mes de marzo presentó los peores resultados de Medidas de Errores de Predicción visto el mes anterior (febrero) que se utiliza para hacer la predicción presentar altos índices de lluvias y nebulosidad.

Las series temporales predichas de la variable $\mathrm{T}$ obtuvieron resultados

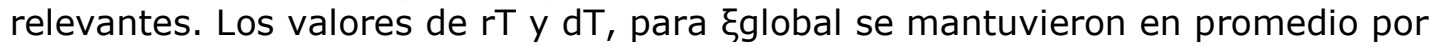
encima de 0,92 en la sequía y por encima de 0,85 en la lluvia; y para छlocal los valores fueron rT y dT por encima de 0,80 en la sequía y por encima de 0,68 en la lluvia. Se resalta también para esta serie temporal, valores pésimos de previsión para el mes de marzo, que por cierto pesaron para bajar la media de los demás parámetros. Excluyendo el mes de marzo, que obtuvo pésima previsión, el promedio de rT y dT para छlocal en la lluvia quedaría por encima de 0,82 . La temperatura es una variable muy importante en el estudio ambiental, ya que es componente de varias otras variables meteorológicas, una buena previsión de estas series indica que otras más pueden ser bien modeladas. Lo que se suma al hecho de ser fácilmente medida en numerosas estaciones meteorológicas.

En cuanto a las series temporales predichas de la variable Rh obtuvieron resultados medianos, los valores de $\mathrm{rRh}$ y $\mathrm{dRh}$, para $\xi g l o b a l$ se mantuvieron cerca de 0,80 en la sequía y por encima de 0,71 en la lluvia, y para छlocal por encima de 0,63 en la sequía y 0,51 en la lluvia. Las predicciones de Rh fueron las que presentaron las menores correlaciones y exacciones y los mayores errores. Esta variable posee una dinámica sensible a la variación de otras variables, principalmente las vinculadas a la circulación atmosférica global. Así como $\mathrm{Rn}$, el mes de marzo para Rh presentó también para esta variable las peores predicciones.

De esta forma, se concluye que el abordaje por teoría de los sistemas dinámicos para análisis y predicción de series temporales no lineales se presenta útil cuando se aplican en datos micrometeorológicos.

\section{REFERENCIAS}

ABARBANEL, H. D. Tools for the analysis of chaotic data. Annals of the New York Academy of Sciences, v.808, n.1, p.1-17, 2006.

ALVES, F. S. M.; FISCH, G.; VENDRAME, I. F. Modificações do microclima e regime hidrológico devido ao desmatamento na Amazônia: um estudo de caso em Rondônia (RO), Brasil. Acta Amazônica, v.29, n.3, p.395-409, 1999. 
ARIEIRA, J.; NUNES DA CUNHA, C. Fitossociologia de uma floresta inundável monodominante de Vochysia divergens Pohl (Vochysiaceae), no Pantanal Norte, MT, Brasil. Acta Botanica Brasilica, v.20, p.569-580, 2006.

BASHARAT, A.; SHAH, M. Time Series Prediction by Chaotic Modeling of Nonlinear Dynamical Systems. IEEE 12th International Conference on Computer Vision. Kyoto, Japão: p.1941-1948, 2009.

BIUDES, M. S.; CAMPELO JUNIOR, J. H.; NOGUEIRA, J. S.; SANCHES, L. Estimativa do balanço de energia em cambarazal e pastagem no norte do Pantanal pelo método da razão de Bowen. Revista Brasileira de Meteorologia, v.24, n.2, p.135-143, 2009.

BIUDES, M. S.; MACHADO, N. G.; DANELICHEN, V. H. M.; SOUZA, M. C.; VOURLITIS, G. L.; NOGUEIRA, J. S. Ground and remote sensing-based measurements of leaf area index in a transitional forest and seasonal flooded forest in Brazil. International Journal of Biometeorology, v.58, n.224, p.1-13, 2013.

BIUDES, M. S.; NOGUEIRA, J. S.; DALMAGRO, H. J.; MACHADO, N. G.; DANELICHEN, V. H. M.; SOUZA, M. C. Mudança no microclima provocada pela conversão de uma floresta de cambará em pastagem no norte do Pantanal. Revista de Ciências Agro-Ambientais, v.10, p.61-68, 2012.

CASDAGLI, M. Nonlinear prediction of chaotic time series. Physica D, v.35, p.335-356, 1989.

CHAUDHURI, S. Predictability of chaos inherent in the occurrence of severe thunderstorms. Advances in Complex Systems, v.9, p.77-85, 2006.

ECKMANN, J. P.; KAMPHORST, S. O.; RUELLE, D. Recurrence plots of dynamical systems. Europhysics Letter, v.4, n.9, p.973-977, 1987.

FERREIRA, H. S. Dinâmica caótica em ecologia: avanços teóricos e metodológicos. Neotropical Biology and Conservation, v.4, n.3, p.125-132, 2009.

FISCH, G. Camada limite Amazônica: aspectos observacionais e de modelagem. 1996. 171f. Tese (Doutorado em Meteorologia) Instituto Nacional de Pesquisas Espaciais, São José dos Campos.

GOMES, A. G.; VARRIALE, M. C. Modelagem de Ecossistemas: Uma Introdução. Editora da Universidade Federal de Santa Maria: Santa Maria, 2004. 503p.

INMET. Normais Climatológicas do Brasil 1961-1990. (Org.) RAMOS, A. M.; SANTOS, L. A. R. ; FORTES, L. T. G. 1. ed. Brasília: 2009. v. 1. 465p.

KANTZ, H.; SCHREIBER, T. Nonlinear Time Series Analysis. Cambridge University Press: Cambridge, UK, 2004. 369 p.

KOEBBE, M.; MAYER-KRESS, G. Use of recurrence plots in the analysis of timeseries data, in: M. Casdagli, S. Eubank (Eds.), Proceedings of SFI Studies in the Science of Complexity, vol. XXI. Redwood City, Addison-Wesley, p.361-378, 1992.

LORENZ, E. N. Deterministic nonperiodic flow. Journal of Atmospheric Sciences, v.20, p.130-141, 1963. 
MARWAN, N.; WESSEL, N.; MEYERFELDT, U.; SCHIRDEWAN, A.; KURTHS, J. Recurrence plot based measures of complexity and its application to heart rate variability data. Physical Review E, v.66, p.026702(1-8), 2002.

MELLO, G. J.; GOMES, R. S. R.; BIUDES, M. S.; DANELICHEN, V. H. M.; MACHADO, N. G. Expoente de Lyapunov de séries de temperatura do Pantanal Norte. In: José de Souza Nogueira; Erondina Azevedo de Lima. (Org.). Coletânea Física Ambiental II. 1. ed. São Paulo: Baraúna, 2012a.

MELLO, G. J. ; GOMES, R. S. R.; BIUDES, M. S.; DANELICHEN, V. H. M.; SOUZA, M. C. Teste de não linearidade de séries temporais micrometeorológicas. In: José de Souza Nogueira; Erondina Azevedo de Lima. (Org.). Coletânea Física Ambiental II. 1. ed. São Paulo: Baraúna, 2012b.

MELLO, G. J.; GOMES, R. S. R.; SILVEIRA, S. W. G.; BIUDES, M. S.; PAULO, S. R. Gráficos de recorrência do balanço de radiação no Pantanal Norte. In: José de Souza Nogueira; Erondina Azevedo de Lima. (Org.). Coletânea Física Ambiental I. 1. ed. São Paulo: Baraúna, 2011.

MELLO, G. J.; PAULO, I. J. C.; PAULO, S. R.; GOMES, R. S. R.; MACHADO, N. G.; NOGUEIRA, J. S.; BIUDES, M. S. Dimensão fractal de séries temporais medidas acima do dossel de floresta no Pantanal Mato-grossense. Revista Brasileira de Climatologia, v.12, p.61-83, 2013b.

MELLO, G. J.; SILVEIRA, S. W. G.; GOMES, R. S. R.; MUSIS, C. R.; BIUDES, M. S.; PAULO, S. R. Análise sazonal da complexidade do saldo de radiação no Pantanal. Revista Ciência e Natura, v.35, p.86-97, $2013 a$.

MILLÁN, H.; GHANBARIAN-ALAVIJEH， B.; GARCÍA-FORNARIS, I. Nonlinear dynamics of mean daily temperature and dewpoint time series at Babolsar, Iran, 1961-2005. Atmospheric Research, v.98, p.89-101, 2010.

MILLÁN, H.; KALAUZI, A.; LLERENA, G.; SUCOSHAÑAY J.; PIEDRA, D. Meteorological complexity in the Amazonian area of Ecuador: An approach based on dynamical system theory. Ecological Complexity, v.6, p.278-285, 2009.

MINDLIN, G. M.; GILMORE, R. Topological analysis and synthesis of chaotic time series. Physica D, v.58, p.229-242, 1992.

NUNES DA CUNHA, C.; JUNK, W. J. Year-to-year changes in water level drive the of Vochysia divergens in Pantanal glassland. Applied Vegetation Science, v.7, p.103-110, 2004.

PINTO, E. G. F.; SAVI, M. A. Nonlinear prediction of time series obtained from an experimental pendulum. Current Topics in Acoustical Research - Research Trends, v.3, p.151-162, 2003.

SAVI, M. A. Dinâmica Não Linear e Caos. Rio de janeiro: Editora E-papers, 2006. $304 \mathrm{p}$.

SCHREIBER, T. Interdisciplinary application of nonlinear time series methods. Physics Reports, v.308, p.1-64, 1999.

THIEL, M.; ROMANO, M. C.; KURTHS, J. How much information is contained in a recurrence plot? Physics Letters A, v.330, n.5, p.343-349, 2004.

TONG, H. Birth of the threshold time series model. Statistica Sinica, v.17, p.8$14,2007$. 
TONG, $\mathrm{H}$. Discussion of 'An analysis of global warming in the Alpine region based on nonlinear nonstationary time series models' by Battaglia and Protopapas. Statistical Methods \& Applications, v.21, n.3, p.335-339, 2012.

TONG, H. Threshold models in nonlinear time series analysis. Lectures Notes in Statistics, vol. 21, Springer, New York, 1983.

TONG, $\mathrm{H}$. Threshold models in time series analysis - 30 years on. Statistics and Its Interface, v.4, p.107-136, 2011.

TSONIS, A. A. The impact of nonlinear dynamicsIn the atmospheric sciences. International Journal of Bifurcation and Chaos, v.11, n.4, p.881-902, 2001.

VIOLA, F. M.; PAIVA, S. L. D.; SAVI, M. A. Analysis of the global warming dynamics from temperature time series. Ecological Modelling, v.221, p.19641978, 2010.

WEBBER Jr., C. L.; ZBILUT, J. P. Dynamical assessment of physiological systems and states using recurrence plot strategies. Journal of Applied Physiology, v.76, p.965-973, 1994.

WEIGEND, A. S.; N. A. GERSHENFELD (Eds.). Time Series Prediction: Forecasting the Future and Understanding the Past. Santa Fe Institute Studies in the Sciences of Complexity XV; Proceedings of the NATO Advanced Research Workshop on Comparative Time Series Analysis. Santa Fe, Addison-Wesley: 1994.

WILLMOTT, C. J. Some comments on the evaluation of model performance. Bulletin American Meteorological Society, v.63, n.11, p.1309-1313, 1982.

WRIGHT, I. R.; NOBRE, C. A.; TOMASELLA, J.; ROCHA, H.R.; ROBERTS, J.M.; VERTAMATTI, E.; CULF, A.D.; ALVALÁ, R.C.; HODNETT, M.G.; UBARANA, V. Towards a GCM surface parameterization for Amazonia. In: GASH, J. H. C.; NOBRE, C. A.; ROBERTS, J. M.; VICTORIA, R. L. (eds.) Amazonian Deforestation and Climate. New York: J. M. Wiley and Sons, 1996, p.473-504.

ZBILUT, J. P.; WEBBER Jr., C. L. Embeddings and delays as derived from quantification of recurrence plots. Physics Letters A, v.171, p.199-203, 1992.

ZBILUT, J. P.; ZALDÍVAR-COMENGES, J. M.; STROZZI, F. Recurrence quantification based Liapunov exponents for monitoring divergence in experimental data. Physics Letters A, v.297, p.173-181, 2002 